\title{
A PRODUCTION INVENTORY MODEL FOR DETERIORATING ITEMS WITH BACKLOG-DEPENDENT DEMAND
}

\author{
Chayanika Rout, Debjani Chakraborty* and Adrijit Goswami
}

\begin{abstract}
This paper investigates a production inventory model under classical EPQ framework with the assumption that the customer demand during the stock-out period is affected by the accumulated back-orders. The backlog rate is not fixed; instead, the demand during stock-out is assumed to decrease proportionally to the existing backlog which is thereby approximated by a piecewise constant function. Deteriorating items are taken into consideration in this work. For better illustration of the theoretical results and to highlight managerial insights, numerical examples are presented which are then compared to the results obtained by considering an exact (non-approximated) backlogging rate (from literature). The comparisons indicate high quality results for the approximated model.
\end{abstract}

Mathematics Subject Classification. 90B05.

Received August 23, 2018. Accepted August 14, 2019.

\section{INTRODUCTION}

Over the decades, production inventory modelling has been one of the most developed fields of operations management. Since Taft [35], one of the pioneers, developed the economic production quantity (EPQ) model as an extension of the economic order quantity (EOQ) model, researchers have extended the basic problem to encounter more realistic situations by relaxing one or several of the underlying assumptions. Mathematical modelling with item depletion has always been an interesting aspect of inventory theory under both EOQ and EPQ frameworks. In general, deterioration may be considered as the result of depletion of stock, which includes damage, decay, spoilage, obsolescence, physical depletion, decreasing usefulness and many more. Foods, volatile liquids, chemicals, pharmaceuticals, blood, drugs, radioactive substances are a few examples of deteriorating items. Deterioration in inventory model was first examined by Ghare and Schrader [14] after which various authors formulated numerous models considering perishable items. The model proposed by Ghare and Schrader with constant perishable rate was later extended by Covert and Philip [10] by introducing variable rate of deterioration. Goswami and Chaudhuri [17] studied an EOQ model for deteriorating items with a linear trend in demand. A heuristic solution procedure was developed by Kim [22] for replenishment of deteriorating items under time-dependent demand rate. In both of the last referred works, the on-hand inventory is assumed to deteriorate at a constant rate. Giri et al. [16] presented a generalized EOQ model with time-dependent deterioration rate. They also assumed the demand rate, holding cost and ordering cost as continuous functions

Keywords. EPQ, deterioration, shortages, backlogging, approximation.

Department of Mathematics, Indian Institute of Technology Kharagpur, 721302 Kharagpur, India.

*Corresponding author: debjani@maths.iitkgp.ac.in 
of time. Widyadana and Wee [41] developed a production inventory model with rework considering replacement for a deteriorated item. It is observed that when the items under consideration are perishable in nature, the demand may need to be backlogged to avoid costs due to deterioration so that the goods can be marketed at a reasonable price. Allowing shortages with partial back-ordering for the case of deteriorating items was pursued by Wee [40], Chang and Dye [7] and Papachristos and Skouri [28]. Wee [40] considered a replenishment policy for items with constant deterioration rate under complete back-ordering of demand. He further extended it to the partial backlogging case also. A supply chain model for perishable items was also developed by Ghiami et al. [15] with stock-dependent demand under partial backlogging and capacity constraints. Yang [44] presented an optimal ordering policy for deteriorating items under time-varying partial backlogging rate. Ahmad and Benkherouf [2] proposed an approach for determining the optimal economic order quantity for the basic EOQ model with backlogging in the presence of non-instantaneous deterioration.

In practical situations, it is observed that the demand of items gets affected by several factors such as quality, service the item provides, advertisement, availability of the item in the market and many more. Over the years, countless models with different patterns of demand rates have come into picture. Recently, stock dependent demand has gained remarkable attention among the researchers. Demand is usually found to be greatly influenced by the stock levels displayed at the customer outlets. Therefore, stocking large amount of inventory not only improves the service level but also stimulates the demand. According to Teng and Chang [36], an increase in shelf space for an item attracts more consumers because of its visibility, popularity or variety. Chung and Wee [9] investigated an integrated production inventory model considering stock-level-dependent demand and Weibull deterioration under partial back-ordering. A lot-sizing model for deteriorating items was developed by Min et al. [25] under current-stock-dependent demand and two-level trade credit. Stavrulaki [34] formulated an inventory management policy of two substitutable products with stock-dependent demand. An inventory model under inflation for perishable items was established by Yang et al. [46] with stock-dependent demand rate under partial back-ordering. Yang [43] demonstrated a profit-maximizing EOQ model with both stock-dependent demand rate and holding cost rate with partial back-orders. Feng et al. [12] proposed a model stipulating the demand in an explicit multivariate function of selling price, freshness and stock displayed. As a matter of fact, backlogging is also observed to affect the demand of consumer goods to quite a good extent. Inventory models with complete and partial backlogging have been extensively studied in the literature. The backlogged demand is either modelled as a function of the existing backlog-size or that of the time remaining to receive the next replenishment (EOQ model) or to resume the production process (EPQ model), as the case may be. It is mainly assumed by the researchers that a shorter waiting time triggers more customers to wait for the fulfilment of the back-orders. So, the rate of backlogged demand increases as the waiting time for the next replenishment (production process) decreases. Chang and Dye [7] modelled a situation where the backlogging rate is dependent upon the length of the waiting time for the next replenishment. Papachristos and Skouri [27] studied a continuous review inventory model for deteriorating items where the rate of backlogged demand increases exponentially as the waiting time for the next replenishment decreases. Some more works in this line of argument may be cited such as Teng et al. [37], Wang [39], Teng et al. [38] and Papachristos and Skouri [28]. A model with waiting time-dependent backlogging rate was also developed by Abad [1] where he assumed the fraction of demand back-ordered to be a decreasing function of the waiting time. Wu et al. [42] proposed an optimal replenishment policy for non-instantaneous deteriorating items with stock-dependent demand and partial back-ordering. He considered a variable backlogging rate dependent upon the waiting time for the next replenishment. An EOQ model with ramp-type demand and Weibull deterioration rate was discussed by Skouri et al. [32] where the backlogging rate is assumed to be any non-increasing function of the waiting time up to the next replenishment. Few more recent research works in this context include the contributions of Kalantari and Taleizadeh [20] and Pal and Adhikari [26].

In many practical situations, it is often observed that the appeal for a product in the market gets reduced due to stock-outs. Refer to goods that satisfy similar need of consumers, for example, tea and coffee, jowar and bajra, groundnut oil and sunflower oil, these are all substitutes to each other. The non-availability of one results in the increase of demand of its substitute which is still displayed on shelves (Breugelmans et al. [3], Campo et al. [5], 
Sloot et al. [33]). It was put forward by Mandelbaum and Zeltyn [24] that the proportion of customers who cease to wait while they are on hold is found to increase with the waiting time. Brown et al. [4] described a survival function that is the fraction of customers who remain on hold after waiting for a given length of time. Gershwin et al. [13] developed a profit maximizing EPQ model accounting to two possible values for the demand rate, namely, high demand and low demand; the switching times being assumed to be random variables with exponential distribution. An inventory model for perishable items was also presented by Duan et al. [11] with stock-dependent demand rate. The authors assumed the backlogging rate to be dependent on the waiting time and the existing backlog size simultaneously. Shah and Naik [30] presented an EOQ model with the assumption that the demand rate declines in proportion to the existing backlog. The authors expressed the customer's demand as a function of time and price. Mahapatra et al. [23] developed an EOQ model for deteriorating items in order to analyse the effect of reliability and time-based demand rate on inventory management system under partial backlogging. An EPQ model with time dependent demand and deterioration rates under partial backordering was studied by Khurana et al. [21]. Skouri [31] proposed an EOQ model with the assumption that the demand rate during the stock-out period is dependent upon the existing amount of shortages and thereby can be approximated by a piecewise constant function. It is to be mentioned in this context that Pentico et al. [29] also approximated an exponential or rational partial back-ordering rate by a constant or linearly changing function of the time remaining until the backorder is fulfilled. Skouri [31] successfully implemented the idea on the basic EOQ framework showing how the approximated backlog rate holds good and provides close results when compared to a model with an exponential backlogging rate without any approximation as used by Gershwin et al. [13]. This has provided us with a good research scope as it can be extended for the case of production inventory model also. Therefore, we aim at filling this research gap in literature with the proposed EPQ model in which deterioration of inventory has also been taken into account.

In this paper, an EPQ model is established in which the demand rate during the stockout period is considered to be a piecewise constant approximation of the back-logged demand function. The remainder of the paper is organised as follows. Section 2 describes the situation of the problem through some fundamental assumptions. Also the notations used throughout this paper are provided in this section. The mathematical model is formulated in Section 3. All the theoretical results are extensively discussed in this section. In Section 4, the proposed model is illustrated with the help of numerical examples with required comparisons. In order to study the implications of changes in parameter values, a complete sensitivity analysis on the total cost is carried out in Section 5. Lastly, a brief conclusion and opportunities for future research are provided in the final section.

\section{PROposed MODEL}

\subsection{Problem description and assumptions}

The following assumptions are used in the model development:

(1) Inventory system with a single type of item is considered over an infinite planning horizon.

(2) Lead time is zero. It is assumed that the raw materials required are supplied instantaneously.

(3) Production rate is constant.

(4) Demand rate is constant when inventory is available in stock.

(5) On-hand inventory deteriorates at a constant rate $\theta$.

(6) Shortages are allowed that affect the demand rate during the stock-out period. When the level of inventory is $x<0$, the demand rate is $\beta(x) D$, where $0 \leq \beta(x) \leq 1$ and $\beta(x)$ is an increasing function of $x(x<0)$. In order to treat a wide variety of $\beta(x)$ functions, $\beta(x)$ is assumed to be piecewise constant as presented below:

$$
\beta(x)= \begin{cases}\beta_{1} & \text { if }-B_{1}<x \leq 0 \\ \beta_{2} & \text { if }-B_{2}<x \leq-B_{1} \\ \beta_{3} & \text { if }-B_{3}<x \leq-B_{2} \\ \cdots & \end{cases}
$$




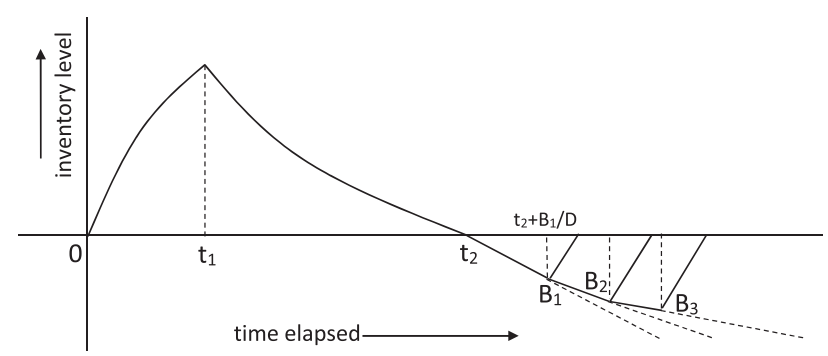

FiguRE 1. Schematic representation of the model.

where $1 \geq \beta_{1} \geq \beta_{2} \geq \beta_{3} \geq 0$ and $0<B_{1}<B_{2}<B_{3}$ (for details, see Skouri [31]).

\subsection{Notations}

The following terminologies are used throughout the work. Some additional notations, wherever required, will be listed accordingly in the paper.

$D$ Demand rate (units per unit time).

$P \quad$ Production rate (units per unit time) $(P>D)$.

$\theta \quad$ Deterioration rate of inventory per unit time $(0<\theta<1)$.

$t_{1} \quad$ Time at which production process stops.

$t_{2} \quad$ Time at which the inventory level drops to zero.

$t^{*} \quad$ Time at which backordering stops and production resumes.

$T$ Cycle time length.

$c \quad$ Setup cost (\$/setup).

$d \quad$ Deterioration cost (\$/unit).

$h \quad$ Holding cost (\$/unit/unit time).

$b \quad$ Backlogging cost (\$/unit/unit time).

$s \quad$ Lost sales cost (\$/unit).

\section{Mathematical Formulation of the MOdel}

A classical EPQ model for deteriorating items is considered under the above-mentioned assumptions. Figure 1 provides a schematic representation of the proposed model. The horizontal axis gives the time elapsed and the inventory level is indicated by the vertical axis. Production starts with zero inventory and continues till $t=t_{1}$ after which the on-hand inventory gets depleted because of both demand and deterioration. At $t=t_{2}$, inventory gets exhausted completely and back-order starts. The production process resumes again at $t=t^{*}$ and the cycle is terminated at $t=T$.

The instantaneous states of inventory level, during a complete cycle of length $T$, can be described by the following set of first order differential equations:

$$
\begin{array}{llrl}
\frac{\mathrm{d} I(t)}{\mathrm{d} t}=P-D-\theta I(t), & 0 \leq t \leq t_{1}, & I(0)=0 . \\
\frac{\mathrm{d} I(t)}{\mathrm{d} t}=-D-\theta I(t), & t_{1} \leq t \leq t_{2}, & I\left(t_{2}\right)=0 . \\
\frac{\mathrm{d} I(t)}{\mathrm{d} t}=-\beta(I(t)) D, & t_{2} \leq t \leq t^{*}, & I\left(t_{2}\right)=0 . \\
\frac{\mathrm{d} I(t)}{\mathrm{d} t}=P-D, & t^{*} \leq t \leq T, & I(T)=0 .
\end{array}
$$


Because of constant demand rate, assumption 6 becomes:

$$
\beta(t)=\left\{\begin{array}{lll}
\beta_{1} & \text { if } \quad t_{2} \leq t \leq t_{2}+\frac{B_{1}}{D} \\
\beta_{2} & \text { if } \quad t_{2}+\frac{B_{1}}{B_{2}} \leq t \leq t_{2}+\frac{B_{2}}{D} \\
\beta_{3} & \text { if } \quad t_{2}+\frac{B_{2}}{D} \leq t
\end{array}\right.
$$

Let $t^{*}$ represent the time at which back-ordering stops and production starts again. Since the total cost is dependent upon the cycle length $T$, so 3 cases are to be considered as follows:

$$
\begin{aligned}
& \text { (A) } t_{2} \leq t^{*} \leq t_{2}+\frac{B_{1}}{D} \\
& \text { (B) } t_{2}+\frac{B_{1}}{D} \leq t^{*} \leq t_{2}+\frac{B_{2}}{D} \\
& \text { (C) } t^{*} \geq t_{2}+\frac{B_{2}}{D}
\end{aligned}
$$

3.1. Case A: $t_{2} \leq t^{*} \leq t_{2}+\frac{B_{1}}{D}$

When $t^{*} \leq t_{2}+\frac{B_{1}}{D}$, the demand rate is $\beta_{1} D$ during the stockout period. So for this case, we have, $\beta_{1} D\left(t^{*}-\right.$ $\left.t_{2}\right)=(P-D)\left(T-t^{*}\right)$ so that

$$
t^{*}=\frac{(P-D) T+\beta_{1} D t_{2}}{P-D+\beta_{1} D}
$$

which implies $t_{2} \leq T \leq t_{2}+\frac{B_{1}}{D}\left(1+\frac{\beta_{1} D}{P-D}\right)$.

During this period, equation (3.2) has the form $\frac{\mathrm{d} I(t)}{\mathrm{d} t}=-\beta_{1} D, \quad t_{2} \leq t \leq t^{*}, \quad I\left(t_{2}\right)=0$.

Therefore, the corresponding solutions of the formulated differential equations are obtained as follows:

$$
\begin{aligned}
I(t) & =\frac{(P-D)}{\theta}\left(1-e^{-\theta t}\right), & & 0 \leq t \leq t_{1} . \\
I(t) & =\frac{D}{\theta}\left[e^{\theta\left(t_{2}-t\right)}-1\right], & & t_{1} \leq t \leq t_{2} . \\
I(t) & =-\beta_{1} D\left(t-t_{2}\right), & & t_{2} \leq t \leq t^{*} . \\
I(t) & =-(P-D)(T-t), & & t^{*} \leq t \leq T .
\end{aligned}
$$

Using the continuity of inventory at $t_{1}$ i.e., $I\left(t_{1}^{-}\right)=I\left(t_{1}^{+}\right)$, we obtain

$$
t_{2}=t_{1}+\frac{1}{\theta} \log \left\{1+\frac{P-D}{D}\left(1-e^{-\theta t_{1}}\right)\right\}
$$


The total inventory cost during the complete cycle comprises of the setup cost, holding cost, deterioration cost, back-order cost and lost sales cost as deduced below:

$$
\begin{aligned}
\text { setup cost per cycle } & =c, \\
\text { holding cost per cycle } & =h\left[\int_{0}^{t_{1}} \frac{P-D}{\theta}\left(1-e^{-\theta t}\right) \mathrm{d} t+\int_{t_{1}}^{t_{2}} \frac{D}{\theta}\left\{e^{\theta\left(t_{2}-t\right)}-1\right\} \mathrm{d} t\right], \\
\text { deterioration cost per cycle } & =d\left[P t_{1}-D t_{2}\right], \\
\text { back-order cost per cycle } & =b\left[\int_{t_{2}}^{t^{*}} \beta_{1} D\left(t-t_{2}\right) \mathrm{d} t+\int_{t^{*}}^{T}(P-D)(T-t) \mathrm{d} t\right] \\
\text { and lost sales cost per cycle } & =s\left(1-\beta_{1}\right) D\left(t^{*}-t_{2}\right) .
\end{aligned}
$$

Summing up all the cost components, the overall inventory cost per unit time is obtained as

$$
\begin{aligned}
\mathrm{TC}_{A}\left(t_{1}, T\right)= & \frac{c}{T}+\frac{h}{\theta^{2} T}\left[(P-D)\left(e^{-\theta t_{1}}-1+\theta t_{1}\right)+D\left\{e^{\theta\left(t_{2}-t_{1}\right)}-1-\theta\left(t_{2}-t_{1}\right)\right\}\right] \\
& +\frac{d}{T}\left(P t_{1}-D t_{2}\right)+\frac{b}{2 T}\left[\beta_{1} D\left(t^{*}-t_{2}\right)^{2}+(P-D)\left(T-t^{*}\right)^{2}\right] \\
& +\frac{s}{T} D\left(1-\beta_{1}\right)\left(t^{*}-t_{2}\right)
\end{aligned}
$$

where $t_{2}$ and $t^{*}$ can be explicitly expressed in terms of $t_{1}$ and $T$.

Therefore the basic optimization problem for this case is

$$
\left\{\begin{array}{l}
\text { Minimize } \mathrm{TC}_{A}\left(t_{1}, T\right) \\
t_{1}>0 \\
t_{2} \leq T \leq t_{2}+\frac{B_{1}}{D}\left(1+\frac{\beta_{1} D}{P-D}\right), \text { where } \\
t_{2}=\frac{P}{D} t_{1}-\theta \frac{P-D}{D} \frac{t_{1}^{2}}{2}
\end{array}\right.
$$

The total cost equation given by (3.5) being highly non-linear in $t_{1}$ and $T$, the partial derivatives are extremely complicated and so it is difficult to analytically obtain the optimal solution. Therefore it is assessed with the help of numerical examples later in our work.

Particular case 1: As, $\theta \rightarrow 0$, we have, $t_{1} \rightarrow \frac{D}{P} t_{2}$ so that $\mathrm{TC}_{A}$ reduces to the following form:

$$
\left.\mathrm{TC}_{A}\right|_{\theta \rightarrow 0}=\frac{c}{T}+\frac{h D(P-D) t_{2}^{2}}{2 T P}+\frac{b \beta_{1} D(P-D)\left(T-t_{2}\right)^{2}}{2 T\left(P-D+\beta_{1} D\right)}+\frac{s D(P-D)\left(1-\beta_{1}\right)\left(T-t_{2}\right)}{T\left(P-D+\beta_{1} D\right)} .
$$

The necessary conditions for minimizing $\mathrm{TC}_{A}\left(t_{2}, T\right)$ are

$$
\begin{aligned}
& \frac{\partial \mathrm{TC}_{A}}{\partial t_{2}}=0 \Longrightarrow t_{2, A}=\frac{b \beta_{1} T+s\left(1-\beta_{1}\right)}{b \beta_{1}+\frac{h}{P}\left(P-D+\beta_{1} D\right)} \quad \text { and } \\
& \frac{\partial \mathrm{TC}_{A}}{\partial T}=0 \Longrightarrow T_{A}=\sqrt{\frac{2 c\left(\frac{P-D+\beta_{1} D}{P-D}\right)\left[b \beta_{1}+\frac{h}{P}\left(P-D+\beta_{1} D\right)\right]-D s^{2}\left(1-\beta_{1}\right)^{2}}{\beta_{1} D b h \frac{P-D+\beta_{1} D}{P}}} \\
& \text { if } 2 c\left(\frac{P-D+\beta_{1} D}{P-D}\right)\left[b \beta_{1}+\frac{h}{P}\left(P-D+\beta_{1} D\right)\right]-D s^{2}\left(1-\beta_{1}\right)^{2}>0 .
\end{aligned}
$$


The sufficient conditions are satisfied by the following:

$$
\begin{aligned}
& \left.\frac{\partial^{2} \mathrm{TC}_{A}\left(t_{2}, T\right)}{\partial t_{2}^{2}}\right|_{t_{2}=t_{2, A}, T=T_{A}}=\frac{D(P-D)}{T_{A}}\left(\frac{h}{P}+\frac{b \beta_{1}}{P-D+\beta_{1} D}\right) \geq 0 \\
& \left.\frac{\partial^{2} \mathrm{TC}_{A}\left(t_{2}, T\right)}{\partial t_{2} \partial T}\right|_{t_{2}=t_{2, A}, T=T_{A}}=-\frac{b \beta_{1} D(P-D)}{T_{A}\left(P-D+\beta_{1} D\right)} \\
& \left.\frac{\partial^{2} \mathrm{TC}_{A}\left(t_{2}, T\right)}{\partial T^{2}}\right|_{t_{2}=t_{2, A}, T=T_{A}}=\frac{b \beta_{1} D(P-D)}{T_{A}\left(P-D+\beta_{1} D\right)} \geq 0 \text { and } \\
& \left.\left.\frac{\partial^{2} \mathrm{TC}_{A}}{\partial t_{2}^{2}}\right|_{t_{2}=t_{2, A}, T=T_{A}} \frac{\partial^{2} \mathrm{TC}_{A}}{\partial T^{2}}\right|_{t_{2}=t_{2, A}, T=T_{A}}-\left(\left.\frac{\partial^{2} \mathrm{TC}_{A}}{\partial t_{2} \partial T}\right|_{t_{2}=t_{2, A}, T=T_{A}}\right)^{2}>0 .
\end{aligned}
$$

So, the deduced expressions for $t_{2, A}$ and $T_{A}$ are optimal which successfully minimize $\left.\mathrm{TC}_{A}\right|_{\theta \rightarrow 0}$.

Results 1. If $2 c\left(\frac{P-D+\beta_{1} D}{P-D}\right)\left[b \beta_{1}+\frac{h}{P}\left(P-D+\beta_{1} D\right)\right]-D s^{2}\left(1-\beta_{1}\right)^{2}>0$, then,

$$
\begin{aligned}
t_{2, A} & =\frac{b \beta_{1} T+s\left(1-\beta_{1}\right)}{b \beta_{1}+\frac{h}{P}\left(P-D+\beta_{1} D\right)} \text { and } \\
T_{A} & =\sqrt{\frac{2 c\left(\frac{P-D+\beta_{1} D}{P-D}\right)\left[b \beta_{1}+\frac{h}{P}\left(P-D+\beta_{1} D\right)\right]-D s^{2}\left(1-\beta_{1}\right)^{2}}{\beta_{1} D b h \frac{P-D+\beta_{1} D}{P}}} \quad \text { provided } \\
t_{2, A} & \leq T_{A} \quad \text { and } \quad T_{A} \leq t_{2, A}+\frac{B_{1}}{D}\left(1+\frac{\beta_{1} D}{P-D}\right)
\end{aligned}
$$

1(a). If $t_{2, A}>T_{A}$, then, $t_{2, A}=T_{A}=\sqrt{\frac{2 c P}{D h(P-D)}}$.

$\mathbf{1}(\mathbf{b})$. If $T_{A}>t_{2, A}+\frac{B_{1}}{D}\left(1+\frac{\beta_{1} D}{P-D}\right)$, then, $t_{2, A}=T_{A}-\frac{B_{1}}{D}\left(1+\frac{\beta_{1} D}{P-D}\right) \quad$ where

$$
\begin{aligned}
T_{A}= & {\left[\frac { 1 } { h D ^ { 2 } ( P - D ) ^ { 2 } } \left(2 c D P(P-D)+2 s B_{1} P D(P-D)\left(1-\beta_{1}\right)+B_{1}^{2}\left(P-D+\beta_{1} D\right)\right.\right.} \\
& \left.\left.\left\{b \beta_{1} P+h\left(P-D+\beta_{1} D\right)\right\}\right)\right]^{1 / 2} .
\end{aligned}
$$

2. If $2 c\left(\frac{P-D+\beta_{1} D}{P-D}\right)\left[b \beta_{1}+\frac{h}{P}\left(P-D+\beta_{1} D\right)\right]-D s^{2}\left(1-\beta_{1}\right)^{2}<0$, then,

$$
t_{2, A}=T_{A}=\sqrt{\frac{2 c P}{D h(P-D)}} .
$$

Similar results are applicable for the deteriorating case also which will be observed to be implemented while solving numerical examples, since an analytical approach for the same could not be achieved. 
Remarks: When $\beta_{1}=1$, we obtain,

$$
t_{2, A}=\frac{b}{b+h} T_{A}=t_{\mathrm{EPQ}} \quad \text { and } \quad T_{A}=\sqrt{\frac{2 c P(b+h)}{b h D(P-D)}}=T_{\mathrm{EPQ}} .
$$

Particular case 2: $\theta \rightarrow 0$ and $P \rightarrow \infty$ gives $t_{1} \rightarrow 0$ and

$$
\mathrm{TC}_{A}=\frac{c}{T}+\frac{h D t_{2}^{2}}{2 T}+\frac{b \beta_{1} D\left(T-t_{2}\right)^{2}}{2 T}+\frac{s D\left(1-\beta_{1}\right)\left(T-t_{2}\right)}{T}
$$

which is in complete agreement with the total cost obtained by Skouri [31] for the EOQ model.

3.2. Case B: $t_{2}+\frac{B_{1}}{D} \leq t^{*} \leq t_{2}+\frac{B_{2}}{D}$

In this case, we have, $\beta_{1} B_{1}+\beta_{2} D\left(t^{*}-t_{2}-\frac{B_{1}}{D}\right)=(P-D)\left(T-t^{*}\right)$ so that

$$
t^{*}=\frac{(P-D) T+\beta_{2} D t_{2}-B_{1}\left(\beta_{1}-\beta_{2}\right)}{P-D+\beta_{2} D}
$$

which implies $t_{2}+\frac{B_{1}}{D}\left(1+\frac{\beta_{1} D}{P-D}\right) \leq T \leq t_{2}+\frac{B_{2}}{D}\left[1+\frac{D}{P-D}\left\{\beta_{2}+\frac{B_{1}}{B_{2}}\left(\beta_{1}-\beta_{2}\right)\right\}\right]$.

During this period, equation (3.2) has the form

$$
\begin{array}{lll}
\frac{\mathrm{d} I(t)}{\mathrm{d} t}=-\beta_{1} D, & t_{2} \leq t \leq t_{2}+\frac{B_{1}}{D}, & I\left(t_{2}\right)=0 \\
\frac{\mathrm{d} I(t)}{\mathrm{d} t}=-\beta_{2} D, & t_{2}+\frac{B_{1}}{D} \leq t \leq t^{*}, & I\left(\left(t_{2}+\frac{B_{1}}{D}\right)^{+}\right)=I\left(\left(t_{2}+\frac{B_{1}}{D}\right)^{-}\right) .
\end{array}
$$

Therefore, the corresponding solutions of the above differential equations are given below:

$$
\begin{array}{ll}
I(t)=-\beta_{1} D\left(t-t_{2}\right), & t_{2} \leq t \leq t_{2}+\frac{B_{1}}{D} . \\
I(t)=-\beta_{2} D\left(t-t_{2}\right)-B_{1}\left(\beta_{1}-\beta_{2}\right), & t_{2}+\frac{B_{1}}{D} \leq t \leq t^{*} .
\end{array}
$$

The setup cost, holding cost and deterioration cost remain the same as obtained in Case A. Apart from these, for this case,

$$
\begin{aligned}
\text { back-order cost per cycle }= & b\left[\int_{t_{2}}^{t_{2}+B_{1} / D} \beta_{1} D\left(t-t_{2}\right) \mathrm{d} t+\int_{t_{2}+B_{1} / D}^{t^{*}} \beta_{2} D\left(t-t_{2}\right) \mathrm{d} t\right. \\
& \left.+\int_{t_{2}+B_{1} / D}^{t^{*}} B_{1}\left(\beta_{1}-\beta_{2}\right) \mathrm{d} t+\int_{t^{*}}^{T}(P-D)(T-t) \mathrm{d} t\right] \\
\text { and lost sales cost per cycle }= & s\left[B_{1}\left(1-\beta_{1}\right)+\left(1-\beta_{2}\right) D\left(t^{*}-t_{2}-\frac{B_{1}}{D}\right)\right] .
\end{aligned}
$$


Summing up all these cost components, the overall inventory cost per unit time takes the following form

$$
\begin{aligned}
\mathrm{TC}_{B}\left(t_{1}, T\right)= & \frac{c}{T}+\frac{h}{\theta^{2} T}\left[(P-D)\left(e^{-\theta t_{1}}-1+\theta t_{1}\right)+D\left\{e^{\theta\left(t_{2}-t_{1}\right)}-1-\theta\left(t_{2}-t_{1}\right)\right\}\right] \\
& +\frac{d}{T}\left(P t_{1}-D t_{2}\right)+\frac{b}{2 T}\left[\frac{\beta_{1} B_{1}^{2}}{D}+\beta_{2} D\left\{\left(t^{*}-t_{2}\right)^{2}-\frac{B_{1}^{2}}{D^{2}}\right\}\right. \\
& \left.+2 B_{1}\left(\beta_{1}-\beta_{2}\right)\left(t^{*}-t_{2}-\frac{B_{1}}{D}\right)+(P-D)\left(T-t^{*}\right)^{2}\right]+\frac{s}{T}\left[B_{1}\left(1-\beta_{1}\right)\right. \\
& \left.+D\left(1-\beta_{2}\right)\left(t^{*}-t_{2}-\frac{B_{1}}{D}\right)\right]
\end{aligned}
$$

Therefore the basic problem for this case is

$$
\left\{\begin{array}{l}
\text { Minimize } \mathrm{TC}_{B}\left(t_{1}, T\right) \\
t_{1}>0 \\
t_{2}+\frac{B_{1}}{D}\left(1+\frac{\beta_{1} D}{P-D}\right) \leq T \leq t_{2}+\frac{B_{2}}{D}\left[1+\frac{D}{P-D}\left\{\beta_{2}+\frac{B_{1}}{B_{2}}\left(\beta_{1}-\beta_{2}\right)\right\}\right], \text { where } \\
t_{2}=\frac{P}{D} t_{1}-\theta \frac{P-D}{D} \frac{t_{1}^{2}}{2}
\end{array}\right.
$$

Particular case 1: As, $\theta \rightarrow 0$, we have, $t_{1} \rightarrow \frac{D}{P} t_{2}$ so that $\mathrm{TC}_{B}$ reduces to the following form:

$$
\begin{aligned}
\left.\mathrm{TC}_{B}\right|_{\theta \rightarrow 0}= & \frac{c}{T}+\frac{h D(P-D)}{2 T P} t_{2}^{2}+\frac{b \beta_{2} D(P-D)}{2 T\left(P-D+\beta_{2} D\right)}\left(T-t_{2}\right)^{2} \\
& -\frac{b B_{1}^{2}\left(\beta_{1}-\beta_{2}\right)}{2 D T} \frac{P-D+\beta_{1} D}{P-D+\beta_{2} D}+\frac{b B_{1}\left(\beta_{1}-\beta_{2}\right)(P-D)}{T\left(P-D+\beta_{2} D\right)}\left(T-t_{2}\right) \\
& +\frac{s D(P-D)\left(1-\beta_{2}\right)}{T\left(P-D+\beta_{2} D\right)}\left(T-t_{2}\right)-\frac{s P B_{1}\left(\beta_{1}-\beta_{2}\right)}{T\left(P-D+\beta_{2} D\right)}
\end{aligned}
$$

$\frac{\partial \mathrm{TC}_{B}}{\partial t_{2}}=0$ and $\frac{\partial \mathrm{TC}_{B}}{\partial T}=0$ respectively build the following relations

$$
\begin{aligned}
t_{2, B}= & \frac{b \beta_{2} D T+b B_{1}\left(\beta_{1}-\beta_{2}\right)+s D\left(1-\beta_{2}\right)}{D\left[b \beta_{2}+\frac{h}{P}\left(P-D+\beta_{2} D\right)\right]} \text { and } \\
T_{B}= & {\left[\frac { P } { \beta _ { 2 } D ^ { 2 } b h ( P - D + \beta _ { 2 } D ) } \left(2 c D\left(\frac{P-D+\beta_{2} D}{P-D}\right)\left\{b \beta_{2}+\frac{h}{P}\left(P-D+\beta_{2} D\right)\right\}\right.\right.} \\
& \left.\left.-s^{2} D^{2}\left(1-\beta_{2}\right)^{2}-b^{2} B_{1}^{2}\left(\beta_{1}-\beta_{2}\right)^{2}-g_{1}\right)\right]^{1 / 2} \text { where, } \\
g_{1}= & B_{1}\left(\beta_{1}-\beta_{2}\right)\left[b B_{1} \frac{P-D+\beta_{1} D}{P-D}\left\{b \beta_{2}+h\left(\frac{P-D+\beta_{2} D}{P}\right)\right\}\right. \\
& \left.+2 s D(h+b)\left(\frac{P-D+\beta_{2} D}{P-D}\right)\right] .
\end{aligned}
$$


The obtained expressions for $t_{2, B}$ and $T_{B}$ are optimal as they satisfy the sufficient conditions for optimality.

Results 1. If $2 c D\left(\frac{P-D+\beta_{2} D}{P-D}\right)\left[b \beta_{2}+\frac{h}{P}\left(P-D+\beta_{2} D\right)\right]-s^{2} D^{2}\left(1-\beta_{2}\right)^{2}-b^{2} B_{1}^{2}\left(\beta_{1}-\beta_{2}\right)^{2}-g_{1}>0$, then,

$$
\begin{aligned}
t_{2, B}= & \frac{b \beta_{2} D T+b B_{1}\left(\beta_{1}-\beta_{2}\right)+s D\left(1-\beta_{2}\right)}{b \beta_{2}+\frac{h}{P}\left(P-D+\beta_{2} D\right)} \text { and } \\
T_{B}= & {\left[\frac { P } { \beta _ { 2 } D ^ { 2 } b h ( P - D + \beta _ { 2 } D ) } \left(2 c D\left(\frac{P-D+\beta_{2} D}{P-D}\right)\left\{b \beta_{2}+\frac{h}{P}\left(P-D+\beta_{2} D\right)\right\}\right.\right.} \\
& \left.\left.-s^{2} D^{2}\left(1-\beta_{2}\right)^{2}-b^{2} B_{1}^{2}\left(\beta_{1}-\beta_{2}\right)^{2}-g_{1}\right)\right]^{1 / 2} \text { provided } \\
t_{2, B} & +\frac{B_{1}}{D}\left(1+\frac{\beta_{1} D}{P-D}\right) \leq T_{B} \leq t_{2, B}+\frac{B_{2}}{D}\left[1+\frac{D}{P-D}\left\{\beta_{2}+\frac{B_{1}}{B_{2}}\left(\beta_{1}-\beta_{2}\right)\right\}\right] .
\end{aligned}
$$

1(a). If $t_{2, B}+\frac{B_{1}}{D}\left(1+\frac{\beta_{1} D}{P-D}\right)>T_{B}$, then, $t_{2, B}=T_{B}-\frac{B_{1}}{D}\left(1+\frac{\beta_{1} D}{P-D}\right) \quad$ where

$$
\begin{aligned}
T_{B}= & {\left[\frac { 1 } { h D ^ { 2 } ( P - D ) ^ { 2 } } \left(2 c D P(P-D)+2 s B_{1} P D(P-D)\left(1-\beta_{1}\right)+B_{1}^{2}\left(P-D+\beta_{1} D\right)\right.\right.} \\
& \left.\left.\left\{b \beta_{1} P+h\left(P-D+\beta_{1} D\right)\right\}\right)\right]^{1 / 2} .
\end{aligned}
$$

1(b). If $T_{B}>t_{2, B}+\frac{B_{2}}{D}\left[1+\frac{D}{P-D}\left\{\beta_{2}+\frac{B_{1}}{B_{2}}\left(\beta_{1}-\beta_{2}\right)\right\}\right]$, then, $t_{2, B}=T_{B}-\frac{B_{2}}{D}$ $\left[1+\frac{D}{P-D}\left\{\beta_{2}+\frac{B_{1}}{B_{2}}\left(\beta_{1}-\beta_{2}\right)\right\}\right]$ where

$$
\begin{aligned}
T_{B}= & {\left[\frac { 1 } { h D ^ { 2 } ( P - D ) ( P - D + \beta _ { 2 } D ) } \left(2 c D P\left(P-D+\beta_{2} D\right)+h B_{2}^{2}(P-D)\left(P-D+\beta_{2} D\right)\right.\right.} \\
& \sigma^{2}+b P \beta_{2} B_{2}^{2}(P-D) \sigma^{2}-b P B_{1}^{2}\left(\beta_{1}-\beta_{2}\right)\left(P-D+\beta_{1} D\right)+2 b P B_{1} B_{2}(P-D)\left(\beta_{1}-\right. \\
& \left.\left.\left.\beta_{2}\right) \sigma+2 s B_{2} P D(P-D)\left(1-\beta_{2}\right) \sigma-2 s D P^{2} B_{1}\left(\beta_{1}-\beta_{2}\right)\right)\right]^{1 / 2}
\end{aligned}
$$

with $\sigma=\left[1+\frac{D}{P-D}\left\{\beta_{2}+\frac{B_{1}}{B_{2}}\left(\beta_{1}-\beta_{2}\right)\right\}\right]$.

2. If $2 c D\left(\frac{P-D+\beta_{2} D}{P-D}\right)\left[b \beta_{2}+\frac{h}{P}\left(P-D+\beta_{2} D\right)\right]-s^{2} D^{2}\left(1-\beta_{2}\right)^{2}-b^{2} B_{1}^{2}\left(\beta_{1}-\beta_{2}\right)^{2}-g_{1}<0$, then, $t_{2, B}=$ $T_{B}-\frac{B_{1}}{D}\left(1+\frac{\beta_{1} D}{P-D}\right) \quad$ where

$$
\begin{aligned}
T_{B}= & {\left[\frac { 1 } { h D ^ { 2 } ( P - D ) ^ { 2 } } \left(2 c D P(P-D)+2 s B_{1} P D(P-D)\left(1-\beta_{1}\right)+B_{1}^{2}\left(P-D+\beta_{1} D\right)\right.\right.} \\
& \left.\left.\left\{b \beta_{1} P+h\left(P-D+\beta_{1} D\right)\right\}\right)\right]^{1 / 2} .
\end{aligned}
$$


Remarks: When $\beta_{1}=\beta_{2}=1$, we obtain,

$$
t_{2, B}=t_{\mathrm{EPQ}} \quad \text { and } \quad T_{B}=T_{\mathrm{EPQ}} .
$$

Particular case 2: $\theta \rightarrow 0$ and $P \rightarrow \infty$ gives $t_{1} \rightarrow 0$ and

$$
\begin{aligned}
\mathrm{TC}_{B}= & \frac{c}{T}+\frac{h D t_{2}^{2}}{2 T}+\frac{b \beta_{2} D\left(T-t_{2}\right)^{2}}{2 T}-\frac{b B_{1}^{2}\left(\beta_{1}-\beta_{2}\right)}{2 D T}+\frac{b B_{1}\left(\beta_{1}-\beta_{2}\right)\left(T-t_{2}\right)}{T} \\
& +\frac{s D\left(1-\beta_{2}\right)\left(T-t_{2}\right)}{T}-\frac{s B_{1}\left(\beta_{1}-\beta_{2}\right)}{T} .
\end{aligned}
$$

3.3. Case C: $t^{*} \geq t_{2}+\frac{B_{2}}{D}$

In this case, we have, $\beta_{1} B_{1}+\beta_{2}\left(B_{2}-B_{1}\right)+\beta_{3} D\left(t^{*}-t_{2}-\frac{B_{2}}{D}\right)=(P-D)\left(T-t^{*}\right)$ so that

$$
t^{*}=\frac{(P-D) T+\beta_{3} D t_{2}-B_{1}\left(\beta_{1}-\beta_{2}\right)-B_{2}\left(\beta_{2}-\beta_{3}\right)}{P-D+\beta_{3} D}
$$

which implies $T \geq t_{2}+\frac{B_{2}}{D}\left[1+\frac{D}{P-D}\left\{\beta_{2}+\frac{B_{1}}{B_{2}}\left(\beta_{1}-\beta_{2}\right)\right\}\right]$.

During this period, equation (3.2) has the form

$$
\begin{array}{llll}
\frac{\mathrm{d} I(t)}{\mathrm{d} t}=-\beta_{1} D, & t_{2} \leq t \leq t_{2}+\frac{B_{1}}{D}, & I\left(t_{2}\right)=0 \\
\frac{\mathrm{d} I(t)}{\mathrm{d} t}=-\beta_{2} D, & t_{2}+\frac{B_{1}}{D} \leq t \leq t_{2}+\frac{B_{2}}{D}, & I\left(\left(t_{2}+\frac{B_{1}}{D}\right)^{+}\right)=I\left(\left(t_{2}+\frac{B_{1}}{D}\right)^{-}\right) \\
\frac{\mathrm{d} I(t)}{\mathrm{d} t}=-\beta_{3} D, & t_{2}+\frac{B_{2}}{D} \leq t \leq t^{*}, & I\left(\left(t_{2}+\frac{B_{2}}{D}\right)^{+}\right)=I\left(\left(t_{2}+\frac{B_{2}}{D}\right)^{-}\right) .
\end{array}
$$

Therefore, the corresponding solutions of the above differential equations are given below:

$$
\begin{array}{ll}
I(t)=-\beta_{1} D\left(t-t_{2}\right), & t_{2} \leq t \leq t_{2}+\frac{B_{1}}{D} . \\
I(t)=-\beta_{2} D\left(t-t_{2}\right)-B_{1}\left(\beta_{1}-\beta_{2}\right), & t_{2}+\frac{B_{1}}{D} \leq t \leq t_{2}+\frac{B_{2}}{D} . \\
I(t)=-\beta_{3} D\left(t-t_{2}\right)-B_{1}\left(\beta_{1}-\beta_{2}\right)-B_{2}\left(\beta_{2}-\beta_{3}\right), & t_{2}+\frac{B_{2}}{D} \leq t \leq t^{*} .
\end{array}
$$

For this case,

$$
\begin{aligned}
\text { back-order cost per cycle }= & b\left[\int_{t_{2}}^{t_{2}+B_{1} / D} \beta_{1} D\left(t-t_{2}\right) \mathrm{d} t+\int_{t_{2}+B_{1} / D}^{t_{2}+B_{2} / D} \beta_{2} D\left(t-t_{2}\right) \mathrm{d} t\right. \\
& +\int_{t_{2}+B_{1} / D}^{t_{2}+B_{2} / D} B_{1}\left(\beta_{1}-\beta_{2}\right) \mathrm{d} t+\int_{t_{2}+B_{2} / D}^{t^{*}} \beta_{3} D\left(t-t_{2}\right) \mathrm{d} t \\
& +\int_{t_{2}+B_{2} / D}^{t^{*}}\left[B_{1}\left(\beta_{1}-\beta_{2}\right)+B_{2}\left(\beta_{2}-\beta_{3}\right)\right] \mathrm{d} t \\
& \left.+\int_{t^{*}}^{T}(P-D)(T-t) \mathrm{d} t\right]
\end{aligned}
$$

and lost sales cost $=s\left[B_{1}\left(1-\beta_{1}\right)+\left(1-\beta_{2}\right)\left(B_{2}-B_{1}\right)+\left(1-\beta_{3}\right) D\left(t^{*}-t_{2}-\frac{B_{2}}{D}\right)\right]$. 
These cost components when are summed up, provide the overall inventory cost per unit time as

$$
\begin{aligned}
\mathrm{TC}_{C}\left(t_{1}, T\right)= & \frac{c}{T}+\frac{h}{\theta^{2} T}\left[(P-D)\left(e^{-\theta t_{1}}-1+\theta t_{1}\right)+D\left\{e^{\theta\left(t_{2}-t_{1}\right)}-1-\theta\left(t_{2}-t_{1}\right)\right\}\right] \\
& +\frac{d}{T}\left(P t_{1}-D t_{2}\right)+\frac{b}{2 T}\left[\frac{\beta_{1} B_{1}^{2}}{D}+\frac{\beta_{2}}{D}\left(B_{2}^{2}-B_{1}^{2}\right)+\frac{2 B_{1}}{D}\left(B_{2}-B_{1}\right)\left(\beta_{1}-\beta_{2}\right)\right. \\
& +\beta_{3} D\left\{\left(t^{*}-t_{2}\right)^{2}-\frac{B_{2}^{2}}{D^{2}}\right\}+2\left\{B_{1}\left(\beta_{1}-\beta_{2}\right)+B_{2}\left(\beta_{2}-\beta_{3}\right)\right\}\left(t^{*}-t_{2}-\frac{B_{2}}{D}\right) \\
& \left.+(P-D)\left(T-t^{*}\right)^{2}\right]+s\left[B_{1}\left(1-\beta_{1}\right)+\left(1-\beta_{2}\right)\left(B_{2}-B_{1}\right)\right. \\
& \left.+D\left(1-\beta_{3}\right)\left(t^{*}-t_{2}-\frac{B_{2}}{D}\right)\right] .
\end{aligned}
$$

Therefore the basic problem for this case is

$$
\left\{\begin{array}{l}
\text { Minimize } \operatorname{TC}_{C}\left(t_{1}, T\right) \\
T \geq 0, \\
\text { where, } \quad t_{2}=\frac{B_{2}}{D}\left[1+\frac{D}{P-D}\left\{\beta_{1}+\theta \frac{P-D}{D} \frac{B_{1}}{B_{2}}\left(\beta_{1}-\beta_{2}\right)\right\}\right]
\end{array}\right.
$$

Particular case 1: As, $\theta \rightarrow 0$, we have, $t_{1} \rightarrow \frac{D}{P} t_{2}$ so that $\mathrm{TC}_{C}$ reduces to the following form:

$$
\begin{aligned}
\left.\mathrm{TC}_{C}\right|_{\theta \rightarrow 0}= & \frac{c}{T}+\frac{h D(P-D) t_{2}^{2}}{2 T P}+\frac{b \beta_{3} D(P-D)\left(T-t_{2}\right)^{2}}{2 T\left(P-D+\beta_{3} D\right)}+\frac{b(P-D)\left(T-t_{2}\right)}{T\left(P-D+\beta_{3} D\right)} \\
& \times\left[B_{1}\left(\beta_{1}-\beta_{2}\right)+B_{2}\left(\beta_{2}-\beta_{3}\right)\right]-\frac{b\left[B_{1}\left(\beta_{1}-\beta_{2}\right)+B_{2}\left(\beta_{2}-\beta_{3}\right)\right]^{2}}{2 T\left(P-D+\beta_{3} D\right)} \\
& -\frac{b}{2 D T}\left[B_{1}^{2}\left(\beta_{1}-\beta_{2}\right)+B_{2}^{2}\left(\beta_{2}-\beta_{3}\right)\right]-\frac{s P\left[B_{1}\left(\beta_{1}-\beta_{2}\right)+B_{2}\left(\beta_{2}-\beta_{3}\right)\right]}{T\left(P-D+\beta_{3} D\right)} \\
& +\frac{s D(P-D)}{T} \frac{\left(1-\beta_{3}\right)\left(T-t_{2}\right)}{P-D+\beta_{3} D} .
\end{aligned}
$$

$\frac{\partial \mathrm{TC}_{C}}{\partial t_{2}}=0$ and $\frac{\partial \mathrm{TC}_{C}}{\partial T}=0$ respectively give

$$
\begin{aligned}
t_{2, C}= & \frac{b \beta_{3} D T+s D\left(1-\beta_{3}\right)+b\left[B_{1}\left(\beta_{1}-\beta_{2}\right)+B_{2}\left(\beta_{2}-\beta_{3}\right)\right]}{D\left[b \beta_{3}+\frac{h}{P}\left(P-D+\beta_{3} D\right)\right]} \text { and } \\
T_{C}= & {\left[\frac { P } { \beta _ { 3 } D ^ { 2 } b h Q } \left(2 c D\left(\frac{Q}{P-D}\right)\left(b \beta_{3}+\frac{h Q}{P}\right)-s^{2} D^{2}\left(1-\beta_{3}\right)^{2}-b^{2} R^{2}\right.\right.} \\
& \left.\left.-2 b \frac{D}{P-D} B_{1} B_{2}\left(\beta_{1}-\beta_{2}\right)\left(\beta_{2}-\beta_{3}\right)\left(b \beta_{3}+\frac{h Q}{P}\right)-g_{1}-g_{2}\right)\right]^{1 / 2} \text { where, } \\
Q= & P-D+\beta_{3} D
\end{aligned}
$$




$$
\begin{aligned}
& R=B_{1}\left(\beta_{1}-\beta_{2}\right)+B_{2}\left(\beta_{2}-\beta_{3}\right), \\
& g_{1}=B_{1}\left(\beta_{1}-\beta_{2}\right)\left[b B_{1}\left(b \beta_{3}+\frac{h Q}{P}\right)\left\{1+\frac{D}{P-D}\left(\beta_{1}-\beta_{2}+\beta_{3}\right)\right\}+2 s D \frac{Q}{P-D}(h+b)\right], \\
& g_{2}=B_{2}\left(\beta_{2}-\beta_{3}\right)\left[b B_{2}\left(b \beta_{3}+\frac{h Q}{P}\right)\left(1+\frac{D \beta_{2}}{P-D}\right)+2 s D \frac{Q}{P-D}(h+b)\right] .
\end{aligned}
$$

The obtained expressions for $t_{2, C}$ and $T_{C}$ are optimal as they satisfy the sufficient conditions for optimality.

Results 1. If $2 c D\left(\frac{Q}{P-D}\right)\left(b \beta_{3}+\frac{h Q}{P}\right)-s^{2} D^{2}\left(1-\beta_{3}\right)^{2}-b^{2} R^{2}+2 b \frac{D}{P-D} B_{1} B_{2}\left(\beta_{1}-\beta_{2}\right)\left(\beta_{2}-\right.$ $\left.\beta_{3}\right)\left(b \beta_{3}+\frac{h Q}{P}\right)-g_{1}-g_{2}>0$, then,

$$
\begin{aligned}
t_{2, C}= & \frac{b \beta_{3} D T+s D\left(1-\beta_{3}\right)+b\left[B_{1}\left(\beta_{1}-\beta_{2}\right)+B_{2}\left(\beta_{2}-\beta_{3}\right)\right]}{D\left[b \beta_{3}+\frac{h}{P}\left(P-D+\beta_{3} D\right)\right]} \text { and } \\
T_{C}= & {\left[\frac { P } { \beta _ { 3 } D ^ { 2 } b h Q } \left(2 c D\left(\frac{Q}{P-D}\right)\left(b \beta_{3}+\frac{h Q}{P}\right)-s^{2} D^{2}\left(1-\beta_{3}\right)^{2}-b^{2} R^{2}\right.\right.} \\
& \left.\left.+2 b \frac{D}{P-D} B_{1} B_{2}\left(\beta_{1}-\beta_{2}\right)\left(\beta_{2}-\beta_{3}\right)\left(b \beta_{3}+\frac{h Q}{P}\right)-g_{1}-g_{2}\right)\right]^{1 / 2}, \text { provided } \\
T_{C} \geq & t_{2, C}+\frac{B_{2}}{D}\left[1+\frac{D}{P-D}\left\{\beta_{2}+\frac{B_{1}}{B_{2}}\left(\beta_{1}-\beta_{2}\right)\right\}\right] .
\end{aligned}
$$

1(a). If $t_{2, C}+\frac{B_{2}}{D}\left[1+\frac{D}{P-D}\left\{\beta_{2}+\frac{B_{1}}{B_{2}}\left(\beta_{1}-\beta_{2}\right)\right\}\right]>T_{C}$, then,

$$
\begin{aligned}
t_{2, C}= & T_{C}-\frac{B_{2}}{D}\left[1+\frac{D}{P-D}\left\{\beta_{2}+\frac{B_{1}}{B_{2}}\left(\beta_{1}-\beta_{2}\right)\right\}\right] \text { where } \\
T_{C}= & {\left[\frac { 1 } { h D ^ { 2 } ( P - D ) ( P - D + \beta _ { 2 } D ) } \left(2 c D P\left(P-D+\beta_{2} D\right)+h B_{2}^{2}(P-D)\left(P-D+\beta_{2} D\right) \sigma^{2}\right.\right.} \\
& +b P \beta_{2} B_{2}^{2}(P-D) \sigma^{2}-b P B_{1}^{2}\left(\beta_{1}-\beta_{2}\right)\left(P-D+\beta_{1} D\right)+2 b P B_{1} B_{2}(P-D)\left(\beta_{1}\right. \\
& \left.\left.\left.-\beta_{2}\right) \sigma+2 s B_{2} P D(P-D)\left(1-\beta_{2}\right) \sigma-2 s D P^{2} B_{1}\left(\beta_{1}-\beta_{2}\right)\right)\right]^{1 / 2} .
\end{aligned}
$$

2. If $2 c D\left(\frac{Q}{P-D}\right)\left(b \beta_{3}+\frac{h Q}{P}\right)-s^{2} D^{2}\left(1-\beta_{3}\right)^{2}-b^{2} R^{2}+2 b \frac{D}{P-D} B_{1} B_{2}\left(\beta_{1}-\beta_{2}\right)\left(\beta_{2}-\beta_{3}\right)\left(b \beta_{3}+\frac{h Q}{P}\right)-$ $g_{1}-g_{2}<0$, then,

$$
\begin{aligned}
t_{2, C}= & T_{C}-\frac{B_{2}}{D}\left[1+\frac{D}{P-D}\left\{\beta_{2}+\frac{B_{1}}{B_{2}}\left(\beta_{1}-\beta_{2}\right)\right\}\right] \text { where } \\
T_{C}= & {\left[\frac { 1 } { h D ^ { 2 } ( P - D ) ( P - D + \beta _ { 2 } D ) } \left(2 c D P\left(P-D+\beta_{2} D\right)+h B_{2}^{2}(P-D)\left(P-D+\beta_{2} D\right)\right.\right.} \\
& \sigma^{2}+b P \beta_{2} B_{2}^{2}(P-D) \sigma^{2}-b P B_{1}^{2}\left(\beta_{1}-\beta_{2}\right)\left(P-D+\beta_{1} D\right)+2 b P B_{1} B_{2}(P-D)\left(\beta_{1}\right. \\
& \left.\left.\left.-\beta_{2}\right) \sigma+2 s B_{2} P D(P-D)\left(1-\beta_{2}\right) \sigma-2 s D P^{2} B_{1}\left(\beta_{1}-\beta_{2}\right)\right)\right]^{1 / 2} .
\end{aligned}
$$


Remarks: When $\beta_{1}=\beta_{2}=\beta_{3}=1$, we obtain,

$$
t_{2, C}=t_{\mathrm{EPQ}} \quad \text { and } \quad T_{C}=T_{\mathrm{EPQ}} .
$$

Particular case 2: $\theta \rightarrow 0$ and $P \rightarrow \infty$ gives $t_{1} \rightarrow 0$ and

$$
\begin{aligned}
\mathrm{TC}_{C}= & \frac{c}{T}+\frac{h D t_{2}^{2}}{2 T}+\frac{b \beta_{3} D\left(T-t_{2}\right)^{2}}{2 T}+\frac{b\left(T-t_{2}\right)}{T}\left[B_{1}\left(\beta_{1}-\beta_{2}\right)+B_{2}\left(\beta_{2}-\beta_{3}\right)\right] \\
& -\frac{b}{2 D T}\left[B_{1}^{2}\left(\beta_{1}-\beta_{2}\right)+B_{2}^{2}\left(\beta_{2}-\beta_{3}\right)\right]-\frac{s\left[B_{1}\left(\beta_{1}-\beta_{2}\right)+B_{2}\left(\beta_{2}-\beta_{3}\right)\right]}{T} \\
& +\frac{s D\left(1-\beta_{3}\right)\left(T-t_{2}\right)}{T} .
\end{aligned}
$$

The total cost of the system can be summarized as:

$$
\mathrm{TC}\left(t_{1}, T\right)=\left\{\begin{array}{rrr}
\mathrm{TC}_{A}\left(t_{1}, T\right), & t_{1}>0, & t_{2} \leq T \leq t_{2}+\frac{B_{1}}{D}\left(1+\frac{\beta_{1} D}{P-D}\right) \\
\mathrm{TC}_{B}\left(t_{1}, T\right), & t_{1}>0, & t_{2}+\frac{B_{1}}{D}\left(1+\frac{\beta_{1} D}{P-D}\right) \leq T \leq t_{2}+\frac{B_{2}}{D}\left[1+\frac{D}{P-D}\right. \\
\quad & \left.\left\{\beta_{2}+\frac{B_{1}}{B_{2}}\left(\beta_{1}-\beta_{2}\right)\right\}\right] \\
\operatorname{TC}_{C}\left(t_{1}, T\right), & t_{1}>0, & T \geq t_{2}+\frac{B_{2}}{D}\left[1+\frac{D}{P-D}\left\{\beta_{2}+\frac{B_{1}}{B_{2}}\left(\beta_{1}-\beta_{2}\right)\right\}\right] .
\end{array}\right.
$$

where $t_{2}=\frac{P}{D} t_{1}-\theta \frac{P-D}{D} \frac{t_{1}^{2}}{2}$.

As already deduced in the respective cases, each branch of $\mathrm{TC}\left(t_{1}, T\right)$ i.e., $\mathrm{TC}_{i}\left(t_{1}, T\right)$ for $i=A, B, C$ is minimized subject to the given constraints. The final optimal solution is attained by comparing the minimum values of $\mathrm{TC}_{i}\left(t_{1}, T\right)(i=A, B, C)$. In order to solve the constrained non-linear minimization problems, we have used the optimization toolbox in MATLAB software (fmincon solver for constrained nonlinear minimization) which uses the well-known Interior Point algorithm. Interior point method is an iterative procedure used in solving both linear and nonlinear convex optimization problems containing inequality constraints. The algorithm satisfies bounds at all iterations and achieves optimization by going through the interior of the feasible space defined by the problem, rather than around its surface.

\section{Numerical Results}

For a better illustration of the theoretical results derived in our proposed work, numerical examples are considered in this section. The results for the approximated model are then compared to those obtained by considering a specific backlogging rate present in the literature. We use the following function from literature (Gershwin et al. [13]) for the exact (without any approximation) backlogging rate

$$
\beta(x)=1-\frac{1}{1+e^{0.5(x+10)}} .
$$

For such backlogging rate, the total inventory cost is calculated as:

$$
\begin{aligned}
\mathrm{TC}_{e}\left(t_{1}, T\right)= & \frac{c}{T}+\frac{h(P-D)}{T}\left(\frac{t_{1}^{2}}{2}-\frac{\theta}{6} t_{1}^{3}\right)+\frac{h D}{T}\left[\frac{(P-D)^{2}}{2 D^{2}}\left(t_{1}^{2}-\theta t_{1}^{3}\right)+\frac{\theta}{6} \frac{(P-D)^{3}}{D^{3}} t_{1}^{3}\right] \\
& +\frac{d \theta}{2 T}\left[(P-D) t_{1}^{2}+\frac{(P-D)^{2}}{D} t_{1}^{2}\right]+\frac{b}{T} \int_{t_{2}}^{t^{*}}|I(t)| \mathrm{d} t+\frac{b}{T} \int_{t^{*}}^{T}(P-D)(T-t) \mathrm{d} t \\
& +\frac{s}{T} \int_{t_{2}}^{t^{*}}\{1-\beta(I(t))\} D \mathrm{~d} t
\end{aligned}
$$


TABLE 1. Results for EPQ model without deterioration using the backlogging rate $\beta(x)=$ $1-\frac{1}{1+e^{0.5(x+10)}}$ and its approximation (Example 1$)$.

\begin{tabular}{|c|c|c|c|c|c|c|}
\hline \multicolumn{2}{|c|}{ Model with } & \multirow{2}{*}{$\begin{array}{l}\beta(x)=1-\frac{1}{1+e^{0.5(x+10)}} \\
\mathrm{TC}_{e}\left(t_{1}, T\right)\end{array}$} & \multicolumn{3}{|c|}{ Model with approximation of $\beta(x)$} & \multirow{2}{*}{$\frac{\mathrm{TC}\left(t_{1}, T\right)-\mathrm{TC}_{e}\left(t_{1}, T\right)}{\mathrm{TC}_{e}\left(t_{1}, T\right)}$} \\
\hline$t_{2}$ & $T$ & & $t_{2}$ & $T$ & $\mathrm{TC}\left(t_{1}, T\right)$ & \\
\hline 0.52 & 0.525 & 779.29 & 0.516 & 0.516 & 774.59 & -0.006 \\
\hline
\end{tabular}

TABLE 2. Results for EPQ model with deterioration using the backlogging rate $\beta(x)=1-$ $\frac{1}{1+e^{0.5(x+10)}}$ and its approximation (Example 1).

\begin{tabular}{|c|c|c|c|c|c|c|}
\hline \multicolumn{2}{|c|}{ Model with } & \multirow{2}{*}{$\frac{(x)=1-\frac{1}{1+e^{0.5(x+10)}}}{\mathrm{TC}_{e}\left(t_{1}, T\right)}$} & \multicolumn{3}{|c|}{ Model with approximation of $\beta(x)$} & \multirow{2}{*}{$\frac{\mathrm{TC}\left(t_{1}, T\right)-\mathrm{TC}_{e}\left(t_{1}, T\right)}{\mathrm{TC}_{e}\left(t_{1}, T\right)}$} \\
\hline$t_{1}$ & $T$ & & $t_{1}$ & $T$ & $\mathrm{TC}\left(t_{1}, T\right)$ & \\
\hline 0.321 & 0.517 & 792.77 & 0.319 & 0.508 & 788.14 & -0.006 \\
\hline
\end{tabular}

where, $I(t)=-2 e^{-5}-D\left(t-t_{2}\right)+2$ Product $\log \left[e^{e^{-5}-5+0.5 D\left(t-t_{2}\right)}\right], \quad t_{2} \leq t \leq t^{*}$.

Following is the data set (Example 1) using which the optimal solution for the proposed model is attained.

$$
\begin{aligned}
D & =1000 \text { units/time, } \\
P & =1600 \text { units/time, } \\
\theta & =0.05, \\
c & =\$ 200, \\
d & =\$ 3 \\
h & =\$ 4 \\
b & =\$ 7 \\
s & =\$ 45 .
\end{aligned}
$$

The backlogging rate expressed in (4.1), as already stated, is approximated by a piecewise constant function given by:

$$
\beta(x)=\left\{\begin{array}{lll}
0.8 & \text { if } \quad-10 \leq x \leq 0 \\
0.5 & \text { if } \quad-20 \leq x \leq-10 \\
0.2 & \text { if } \quad x \leq-20
\end{array}\right.
$$

The exact backlogging rate $\beta(x)$ provided in (4.1) and its piecewise constant approximation represented by (4.2) are graphically illustrated in the work of Skouri [31].

The results obtained numerically for both the exact and approximate models are presented in Tables 1 (model without any deterioration) and 2 (model considering deteriorating items). The optimal values for the decision variables along with the relative changes in the inventory costs for both the models are thereby tabulated. 
TABLE 3. Results for EPQ model without deterioration using the backlogging rate $\beta(x)=$ $1-\frac{1}{1+e^{0.5(x+10)}}$ and its approximation (Example 2).

\begin{tabular}{|c|c|c|c|c|c|c|}
\hline \multicolumn{2}{|c|}{ Model with } & $x)=1-\frac{1}{1+e^{0.5(x+10)}}$ & \multicolumn{3}{|c|}{ Model with approximation of $\beta(x)$} & \multirow{2}{*}{$\frac{\mathrm{TC}\left(t_{1}, T\right)-\mathrm{TC}_{e}\left(t_{1}, T\right)}{\mathrm{TC}_{e}\left(t_{1}, T\right)}$} \\
\hline$t_{2}$ & $T$ & $\mathrm{TC}_{e}\left(t_{1}, T\right)$ & $t_{2}$ & $T$ & $\mathrm{TC}\left(t_{1}, T\right)$ & \\
\hline 3.622 & 4.952 & 417.29 & 3.856 & 4.395 & 444.21 & +0.06 \\
\hline
\end{tabular}

TABLE 4. Results for EPQ model with deterioration using the backlogging rate $\beta(x)=1-$ $\frac{1}{1+e^{0.5(x+10)}}$ and its approximation (Example 2).

\begin{tabular}{|c|c|c|c|c|c|c|}
\hline \multicolumn{3}{|c|}{ Model with $\beta(x)=1-\frac{1}{1+e^{0.5(x+10)}}$} & \multicolumn{3}{|c|}{ Model with approximation of $\beta(x)$} & \multirow[t]{2}{*}{$\frac{\mathrm{TC}\left(t_{1}, T\right)-\mathrm{TC}_{e}\left(t_{1}, T\right)}{\mathrm{TC}_{e}\left(t_{1}, T\right)}$} \\
\hline$t_{1}$ & $T$ & $\mathrm{TC}_{e}\left(t_{1}, T\right)$ & $t_{1}$ & $T$ & $\mathrm{TC}\left(t_{1}, T\right)$ & \\
\hline 2.387 & 4.957 & 420.18 & 2.553 & 4.397 & 447.66 & +0.07 \\
\hline
\end{tabular}

As a second example (Example 2), certain parameters in Example 1 are modified as follows:

$$
\begin{aligned}
D & =80 \text { units } / \text { time } \\
P & =125 \text { units } / \text { time }, \\
c & =\$ 1000, \\
s & =\$ 10 .
\end{aligned}
$$

Rest of the parameters are kept unchanged. Tables 3 and 4 illustrate the numerical results for the second example.

In Example 1, the optimal solutions for the approximated model are attained by the application of Result 2 as described in Section 3.1. Similarly, for Example 2, the same is achieved by using Result 1(b) depicted in Section 3.2.

Therefore it is quite evident from the tabulated results, that the approximation for the backlogging rate is proved to perform really well when compared to the exact model. And this is true in terms of both the decision variables and the total cost.

\section{SENSITIVITY ANALYSIS}

In any decision-making process, it is quite evident that the values of the parameters do not always remain fixed. Variations in their values may inevitably appear due to dynamic market condition and other uncertainties. So, in order to provide better managerial insights, the sensitivity analysis is found to be of great help to encounter the possible implications of the corresponding changes.

In this section, sensitivity analysis of the various parameters involved has been conducted for both the exact and approximate models and the results are presented in Tables 5 and 6 for Example 1 and in Tables 7 and 8 for Example 2. The value of only one parameter is altered at a time keeping all the other parameters unchanged and the effects of such variations are observed. The said changes are carried out with a decrement in the parameter value by $30 \%$ and $15 \%$ and then with an increment by $15 \%$ and $30 \%$.

Based upon the results presented in Tables 5 and 7, the interpretations are as follows: 
TABLE 5. Sensitivity analysis for model with deterioration (Example 1).

\begin{tabular}{|c|c|c|c|c|c|c|c|c|}
\hline \multirow{2}{*}{$\begin{array}{l}\text { Par } \\
(i)\end{array}$} & \multirow{2}{*}{$\begin{array}{l}\text { \%age } \\
\text { change }\end{array}$} & \multicolumn{3}{|c|}{ Model with $\beta(x)=1-\frac{1}{1+e^{0.5(x+10)}}$} & \multicolumn{3}{|c|}{ Model with approximated $\beta(x)$} & \multirow[t]{2}{*}{$\frac{\mathrm{TC}-\mathrm{TC}_{e}}{\mathrm{TC}_{e}}$} \\
\hline & & $t_{1}$ & $T$ & $\mathrm{TC}_{e}\left(t_{1}, T\right)$ & $t_{1}$ & $T$ & $\mathrm{TC}\left(t_{1}, T\right)$ & \\
\hline \multirow[t]{4}{*}{$P$} & -30 & 0.859 & 0.999 & 418.90 & 0.860 & 0.961 & 419.10 & +0.0005 \\
\hline & -15 & 0.451 & 0.620 & 665.56 & 0.448 & 0.606 & 661.30 & -0.006 \\
\hline & +15 & 0.252 & 0.466 & 874.44 & 0.251 & 0.460 & 870.23 & -0.005 \\
\hline & +30 & 0.209 & 0.436 & 932.14 & 0.208 & 0.431 & 928.52 & -0.004 \\
\hline \multirow[t]{4}{*}{$D$} & -30 & 0.218 & 0.501 & 810.02 & 0.218 & 0.494 & 808.88 & -0.001 \\
\hline & -15 & 0.264 & 0.500 & 816.19 & 0.263 & 0.492 & 813.05 & -0.004 \\
\hline & +15 & 0.398 & 0.559 & 736.65 & 0.395 & 0.548 & 731.27 & -0.007 \\
\hline & +30 & 0.520 & 0.648 & 638.93 & 0.516 & 0.633 & 634.01 & -0.008 \\
\hline \multirow[t]{4}{*}{$\theta$} & -30 & 0.322 & 0.519 & 788.75 & 0.320 & 0.511 & 784.11 & -0.006 \\
\hline & -15 & 0.321 & 0.518 & 790.76 & 0.320 & 0.509 & 786.13 & -0.006 \\
\hline & +15 & 0.320 & 0.515 & 794.76 & 0.319 & 0.507 & 790.15 & -0.006 \\
\hline & +30 & 0.320 & 0.514 & 796.75 & 0.318 & 0.506 & 792.15 & -0.006 \\
\hline \multirow[t]{4}{*}{$c$} & -30 & 0.269 & 0.433 & 666.46 & 0.267 & 0.425 & 659.52 & -0.01 \\
\hline & -15 & 0.296 & 0.477 & 732.38 & 0.294 & 0.468 & 726.69 & -0.008 \\
\hline & +15 & 0.344 & 0.554 & 848.82 & 0.342 & 0.545 & 845.12 & -0.004 \\
\hline & +30 & 0.365 & 0.588 & 901.37 & 0.364 & 0.579 & 898.48 & -0.003 \\
\hline \multirow[t]{4}{*}{$d$} & -30 & 0.323 & 0.519 & 788.50 & 0.321 & 0.511 & 783.85 & -0.006 \\
\hline & -15 & 0.322 & 0.518 & 790.64 & 0.320 & 0.509 & 786.00 & -0.006 \\
\hline & +15 & 0.320 & 0.515 & 794.89 & 0.318 & 0.507 & 790.28 & -0.006 \\
\hline & +30 & 0.319 & 0.514 & 797.01 & 0.317 & 0.505 & 792.41 & -0.006 \\
\hline \multirow[t]{4}{*}{$h$} & -30 & 0.382 & 0.611 & 669.24 & 0.379 & 0.603 & 664.36 & -0.007 \\
\hline & -15 & 0.347 & 0.558 & 733.72 & 0.345 & 0.549 & 728.88 & -0.007 \\
\hline & +15 & 0.300 & 0.484 & 847.52 & 0.298 & 0.475 & 843.25 & -0.005 \\
\hline & +30 & 0.282 & 0.456 & 898.75 & 0.281 & 0.447 & 894.98 & -0.004 \\
\hline \multirow[t]{4}{*}{$b$} & -30 & 0.321 & 0.517 & 792.71 & 0.319 & 0.508 & 788.14 & -0.006 \\
\hline & -15 & 0.321 & 0.517 & 792.74 & 0.319 & 0.508 & 788.14 & -0.006 \\
\hline & +15 & 0.321 & 0.517 & 792.79 & 0.319 & 0.508 & 788.14 & -0.006 \\
\hline & +30 & 0.321 & 0.517 & 792.82 & 0.319 & 0.508 & 788.14 & -0.006 \\
\hline \multirow[t]{4}{*}{$s$} & -30 & 0.319 & 0.517 & 788.02 & 0.319 & 0.508 & 788.14 & +0.0002 \\
\hline & -15 & 0.320 & 0.517 & 790.63 & 0.319 & 0.508 & 788.14 & -0.003 \\
\hline & +15 & 0.322 & 0.517 & 794.57 & 0.319 & 0.508 & 788.14 & -0.008 \\
\hline & +30 & 0.322 & 0.516 & 796.13 & 0.319 & 0.508 & 788.14 & -0.01 \\
\hline
\end{tabular}

(1) The backlogging rate $\beta(x)$ performs very well as a piecewise constant approximation of the exact rate present in the literature in terms of both the decision variables and the overall cost.

(2) The outcomes indicate that both the models are more sensitive to changes in the values of production rate, demand rate, setup cost, holding cost and moderately sensitive to the remaining parameters.

(3) The increment in the overall cost has a direct relation to the increment in the production rate as well as the rate of deterioration.

(4) As the demand rate rises while the other parameters are kept unchanged, the total cost is found to decrease. This can be accounted due to decrease in both the holding and deterioration costs.

The relative changes in the decision variables and total cost are also observed due to variations in the factors estimating the approximated backlogging rate. The corresponding outcomes are presented in Tables 6 and 8 for Examples 1 and 2 respectively. It is observed that as the value of the parameter $\beta_{1}$ is increased by $30 \%$, its value exceeds 1 . But since $\beta_{1}$ should lie between 0 and 1 , so the computations for this case are not taken into account. 
TABLE 6. Sensitivity analysis for model with deterioration (Example 1).

\begin{tabular}{|c|c|c|c|c|c|}
\hline \multirow{2}{*}{$\begin{array}{l}\text { Parameter } \\
(i)\end{array}$} & \multirow{2}{*}{$\begin{array}{l}\text { Percentage } \\
\text { change }(\%)\end{array}$} & \multicolumn{3}{|c|}{ Model with approximation of $\beta(x)$} & \multirow[t]{2}{*}{$\frac{\mathrm{TC}\left(t_{1}, T\right)-\mathrm{TC}_{e}\left(t_{1}, T\right)}{\mathrm{TC}_{e}\left(t_{1}, T\right)}$} \\
\hline & & $t_{1}$ & $T$ & $\mathrm{TC}\left(t_{1}, T\right)$ & \\
\hline \multirow[t]{4}{*}{$B_{1}$} & -30 & 0.319 & 0.508 & 788.14 & -0.006 \\
\hline & -15 & 0.319 & 0.508 & 788.14 & -0.006 \\
\hline & +15 & 0.319 & 0.508 & 788.14 & -0.006 \\
\hline & +30 & 0.319 & 0.508 & 788.14 & -0.006 \\
\hline \multirow[t]{4}{*}{$B_{2}$} & -30 & 0.319 & 0.508 & 788.14 & -0.006 \\
\hline & -15 & 0.319 & 0.508 & 788.14 & -0.006 \\
\hline & +15 & 0.319 & 0.508 & 788.14 & -0.006 \\
\hline & +30 & 0.319 & 0.508 & 788.14 & -0.006 \\
\hline \multirow[t]{4}{*}{$\beta_{1}$} & -30 & 0.319 & 0.508 & 788.14 & -0.006 \\
\hline & -15 & 0.318 & 0.508 & 788.14 & -0.006 \\
\hline & +15 & 0.318 & 0.508 & 788.14 & -0.006 \\
\hline & +30 & - & - & - & - \\
\hline \multirow[t]{4}{*}{$\beta_{2}$} & -30 & 0.319 & 0.508 & 788.14 & -0.006 \\
\hline & -15 & 0.319 & 0.508 & 788.14 & -0.006 \\
\hline & +15 & 0.319 & 0.508 & 788.14 & -0.006 \\
\hline & +30 & 0.319 & 0.508 & 788.14 & -0.006 \\
\hline \multirow[t]{4}{*}{$\beta_{3}$} & -30 & 0.319 & 0.508 & 788.14 & -0.006 \\
\hline & -15 & 0.319 & 0.508 & 788.14 & -0.006 \\
\hline & +15 & 0.319 & 0.508 & 788.14 & -0.006 \\
\hline & +30 & 0.319 & 0.508 & 788.14 & -0.006 \\
\hline
\end{tabular}

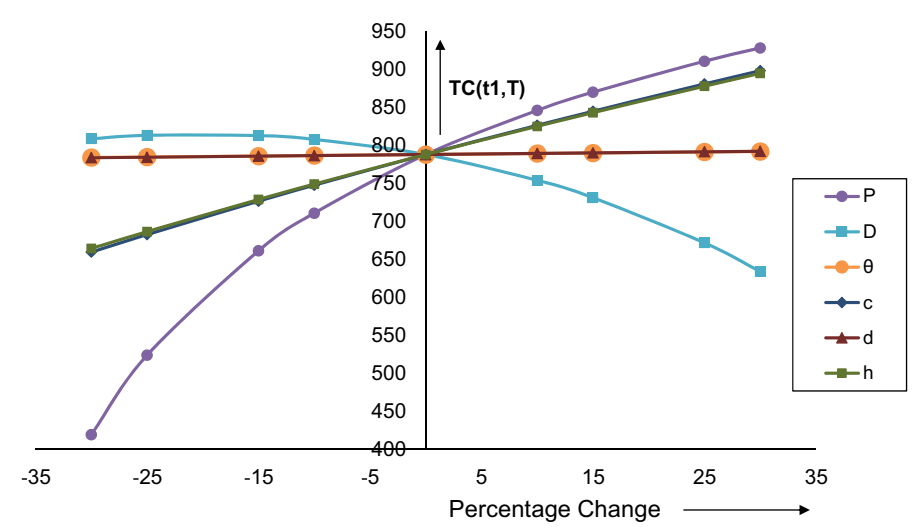

Figure 2. Graphical plot showing variation in $\mathrm{TC}\left(t_{1}, T\right)$ due to changes in values of certain parameters (Example 1).

An important conclusion can be drawn from the tabulated outcomes that any change in the approximation of the exact backlogging rate function hardly affects the optimal cost and decision variables.

The graphical illustrations for some highly sensitive parameters corresponding to Examples 1 and 2 are respectively shown in Figures 2 and 3. It is to be noted that the approximated backlogging rate has been observed to be noticeably good for both underestimation and overestimation of the model parameters. 
TABLE 7. Sensitivity analysis for model with deterioration (Example 2).

\begin{tabular}{|c|c|c|c|c|c|c|c|c|}
\hline \multirow{2}{*}{$\begin{array}{l}\text { Par } \\
\text { (i) }\end{array}$} & \multirow{2}{*}{$\begin{array}{l}\text { \%age } \\
\text { change }\end{array}$} & \multicolumn{3}{|c|}{ Model with $\beta(x)=1-\frac{1}{1+e^{0.5(x+10)}}$} & \multicolumn{3}{|c|}{ Model with approximated $\beta(x)$} & \multirow[t]{2}{*}{$\frac{\mathrm{TC}-\mathrm{TC}_{e}}{\mathrm{TC}_{e}}$} \\
\hline & & $t_{1}$ & $T$ & $\mathrm{TC}_{e}\left(t_{1}, T\right)$ & $t_{1}$ & $T$ & $\mathrm{TC}\left(t_{1}, T\right)$ & \\
\hline \multirow[t]{4}{*}{$P$} & -30 & 7.194 & 11.128 & 188.06 & 7.660 & 10.231 & 198.06 & +0.05 \\
\hline & -15 & 3.378 & 6.133 & 338.60 & 3.642 & 5.450 & 362.68 & +0.07 \\
\hline & +15 & 1.880 & 4.397 & 474.75 & 1.995 & 3.908 & 502.44 & +0.06 \\
\hline & +30 & 1.562 & 4.063 & 514.49 & 1.647 & 3.622 & 541.26 & +0.05 \\
\hline \multirow[t]{4}{*}{$D$} & -30 & 1.612 & 4.693 & 443.50 & 1.698 & 4.154 & 466.06 & +0.05 \\
\hline & -15 & 1.957 & 4.721 & 440.99 & 2.077 & 4.188 & 466.60 & +0.06 \\
\hline & +15 & 2.984 & 5.485 & 379.65 & 3.217 & 4.867 & 406.96 & +0.07 \\
\hline & +30 & 3.980 & 6.616 & 314.55 & 4.313 & 5.893 & 338.10 & +0.07 \\
\hline \multirow[t]{4}{*}{$\theta$} & -30 & 2.365 & 4.954 & 419.37 & 2.527 & 4.394 & 446.70 & +0.07 \\
\hline & -15 & 2.376 & 4.955 & 419.78 & 2.540 & 4.396 & 447.18 & +0.07 \\
\hline & +15 & 2.398 & 4.959 & 420.57 & 2.567 & 4.399 & 448.11 & +0.07 \\
\hline & +30 & 2.409 & 4.961 & 420.94 & 2.581 & 4.402 & 448.55 & +0.07 \\
\hline \multirow[t]{4}{*}{$c$} & -30 & 1.990 & 4.076 & 353.72 & 2.110 & 3.745 & 373.95 & +0.06 \\
\hline & -15 & 2.197 & 4.534 & 388.57 & 2.339 & 4.083 & 412.28 & +0.06 \\
\hline & +15 & 2.563 & 5.351 & 449.28 & 2.755 & 4.692 & 480.66 & +0.07 \\
\hline & +30 & 2.729 & 5.722 & 476.38 & 2.947 & 4.971 & 511.71 & +0.07 \\
\hline \multirow[t]{4}{*}{$d$} & -30 & 2.404 & 4.975 & 418.49 & 2.570 & 4.421 & 445.50 & +0.06 \\
\hline & -15 & 2.396 & 4.966 & 419.34 & 2.562 & 4.409 & 446.58 & +0.06 \\
\hline & +15 & 2.378 & 4.948 & 421.01 & 2.545 & 4.385 & 448.73 & +0.07 \\
\hline & +30 & 2.370 & 4.939 & 421.85 & 2.537 & 4.373 & 449.80 & +0.07 \\
\hline \multirow[t]{4}{*}{$h$} & -30 & 2.985 & 5.597 & 368.13 & 3.125 & 5.229 & 384.07 & +0.04 \\
\hline & -15 & 2.647 & 5.230 & 396.14 & 2.801 & 4.759 & 417.54 & +0.05 \\
\hline & +15 & 2.178 & 4.747 & 441.10 & 2.356 & 4.108 & 475.12 & +0.08 \\
\hline & +30 & 2.006 & 4.579 & 459.50 & 2.195 & 3.870 & 500.43 & +0.09 \\
\hline \multirow[t]{4}{*}{$b$} & -30 & 2.303 & 5.269 & 406.25 & 2.543 & 4.381 & 445.89 & +0.09 \\
\hline & -15 & 2.350 & 5.087 & 414.08 & 2.548 & 4.389 & 446.77 & +0.08 \\
\hline & +15 & 2.417 & 4.860 & 425.10 & 2.559 & 4.405 & 448.54 & +0.06 \\
\hline & +30 & 2.441 & 4.786 & 429.17 & 2.564 & 4.413 & 449.42 & +0.05 \\
\hline \multirow[t]{4}{*}{$s$} & -30 & 2.333 & 5.055 & 411.19 & 2.524 & 4.354 & 442.86 & +0.08 \\
\hline & -15 & 2.361 & 5.007 & 415.87 & 2.539 & 4.376 & 445.26 & +0.07 \\
\hline & +15 & 2.411 & 4.907 & 424.13 & 2.568 & 4.418 & 450.04 & +0.06 \\
\hline & +30 & 2.432 & 4.856 & 427.73 & 2.582 & 4.439 & 452.41 & +0.06 \\
\hline
\end{tabular}

\section{Concluding REMARKS}

In the proposed work, a production inventory model for deteriorating items is discussed showing how the customer demand is affected by shortages. A piecewise constant approximation of the backlogged demand is considered. Following the analytical approach, closed form solutions for the decision variables are obtained for the non-deteriorating case as illustrated in Section 3. Whereas a numerical optimization technique is employed to obtain solutions for the deteriorating case because of its operational complexity. Similar results are established for both the exact and approximate models such that the approximated function for the backlog-rate is proved to be good in this regard. It is clear from the sensitivity analysis conducted that the total cost incurred tends to rise with the increment in the deterioration rate of the items whereas it remains almost unaffected due to any misestimation of the backlogging rate approximation.

The model is developed in a completely crisp environment thereby providing an opportunity to the researchers to extend the same to stochastic or fuzzy environments in order to encounter even more practical scenarios. As 
TABLE 8. Sensitivity analysis for model with deterioration (Example 2).

\begin{tabular}{|c|c|c|c|c|c|}
\hline \multirow{2}{*}{$\begin{array}{l}\text { Parameter } \\
(i)\end{array}$} & \multirow{2}{*}{$\begin{array}{l}\text { Percentage } \\
\text { change }(\%)\end{array}$} & \multicolumn{3}{|c|}{ Model with approximation of $\beta(x)$} & \multirow[t]{2}{*}{$\frac{\mathrm{TC}\left(t_{1}, T\right)-\mathrm{TC}_{e}\left(t_{1}, T\right)}{\mathrm{TC}_{e}\left(t_{1}, T\right)}$} \\
\hline & & $t_{1}$ & $T$ & $\mathrm{TC}\left(t_{1}, T\right)$ & \\
\hline \multirow[t]{4}{*}{$B_{1}$} & -30 & 2.575 & 4.408 & 451.14 & +0.07 \\
\hline & -15 & 2.564 & 4.403 & 449.40 & +0.07 \\
\hline & +15 & 2.543 & 4.392 & 445.91 & +0.06 \\
\hline & +30 & 2.532 & 4.386 & 444.16 & +0.06 \\
\hline \multirow[t]{4}{*}{$B_{2}$} & -30 & 2.585 & 4.301 & 452.80 & +0.08 \\
\hline & -15 & 2.568 & 4.348 & 450.11 & +0.07 \\
\hline & +15 & 2.540 & 4.448 & 445.44 & +0.06 \\
\hline & +30 & 2.528 & 4.501 & 443.44 & +0.06 \\
\hline \multirow[t]{4}{*}{$\beta_{1}$} & -30 & 2.609 & 4.426 & 456.85 & +0.09 \\
\hline & -15 & 2.581 & 4.411 & 452.22 & +0.08 \\
\hline & +15 & 2.526 & 4.384 & 443.15 & +0.05 \\
\hline & +30 & - & - & - & - \\
\hline \multirow[t]{4}{*}{$\beta_{2}$} & -30 & 2.590 & 4.417 & 453.67 & +0.08 \\
\hline & -15 & 2.572 & 4.407 & 450.65 & +0.07 \\
\hline & +15 & 2.535 & 4.387 & 444.69 & +0.06 \\
\hline & +30 & 2.517 & 4.378 & 441.74 & +0.05 \\
\hline \multirow[t]{4}{*}{$\beta_{3}$} & -30 & 2.553 & 4.397 & 447.66 & +0.07 \\
\hline & -15 & 2.553 & 4.397 & 447.66 & +0.07 \\
\hline & +15 & 2.553 & 4.397 & 447.66 & +0.07 \\
\hline & +30 & 2.553 & 4.397 & 447.66 & +0.07 \\
\hline
\end{tabular}

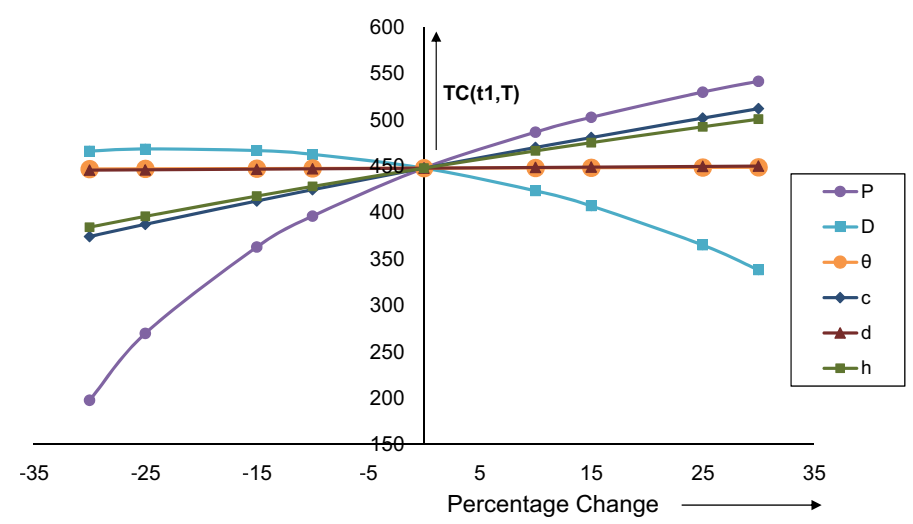

FiguRE 3. Graphical plot showing variation in $\mathrm{TC}\left(t_{1}, T\right)$ due to changes in values of certain parameters (Example 2).

other possible extensions of this model, an integrated vendor-buyer approach (Huang [19], Yang and Wee [45]), imperfections in the production process taking into account both Type 1 and Type 2 inspection errors as suggested in the work of Yoo et al. [47] can be considered. Considering multi items with promotional efforts (Cardenas-Barron and Sana [6]) can also be considered as a good scope for future research. Moreover, currently one of the major concerns of the companies is to reduce carbon emissions associated with their operations. 
So, based on some recent findings by Chen et al. [8] and Hovelaque and Bironneau [18], carbon emission constraints can also be imposed into the proposed model.

Acknowledgements. The authors would like to acknowledge the support provided by the Indian Institute of Technology Kharagpur yielding facilities for research. The authors would also like to thank the Editors and the anonymous referees for their valuable comments and suggestions on improving this paper.

\section{REFERENCES}

[1] P.L. Abad, Optimal pricing and lot-sizing under conditions of perishability, finite production and partial backordering and lost sale. Eur. J. Oper. Res. 144 (2003) 677-685.

[2] B. Ahmad and L. Benkherouf, Economic-order-type inventory models for non-instantaneous deteriorating items and backlogging. RAIRO: OR 52 (2018) 895-901.

[3] E. Breugelmans, K. Campo and E. Gijsbrechts, Opportunities for active stock-out management in online stores: the impact of the stock-out policy on online stock-out reactions. J. Retail. 82 (2006) 215-228.

[4] L. Brown, N. Gans, A. Mandelbaum, A. Sakov, S. Zeltyn, L. Zhao and S. Haipeng, Statistical analysis of a telephone call center: a queueing-science perspective. J. Am. Stat. Assoc. 100 (2005) 36-50.

[5] K. Campo, E. Gijsbrechts and P. Nisol, Towards understanding consumer response to stock-outs. J. Retail. 76 (2000) $219-242$.

[6] L.E. Cardenas-Barron and S.S. Sana, Multi-item EOQ inventory model in a two-layer supply chain while demand varies with promotional effort. Appl. Math. Modell. 39 (2015) 6725-6737.

[7] H.-J. Chang and C.-Y. Dye, An EOQ model for deteriorating items with time varying demand and partial backlogging. J. Oper. Res. Soc. 50 (1999) 1176-1182.

[8] X. Chen, S. Benjaafar and A. Elomri, The carbon-constrained EOQ. Oper. Res. Lett. 41 (2013) 172-179.

[9] C.J. Chung and H.-M. Wee, An integrated production-inventory deteriorating model for pricing policy considering imperfect production, inspection planning and warranty-period-and stock-level-dependant demand. Int. J. Syst. Sci. 39 (2008) $823-837$.

[10] R.P. Covert and G.C. Philip, An EOQ model for items with weibull distribution deterioration. AIIE Trans. 5 (1973) $323-326$.

[11] Y. Duan, G. Li, J.M. Tien and J. Huo, Inventory models for perishable items with inventory level dependent demand rate. Appl. Math. Modell. 36 (2012) 5015-5028.

[12] L. Feng, Y.-L. Chan and L.E. Cardenas-Barron, Pricing and lot-sizing polices for perishable goods when the demand depends on selling price, displayed stocks, and expiration date. Int. J. Prod. Econ. 185 (2017) 11-20.

[13] S.B. Gershwin, B. Tan and M.H. Veatch, Production control with backlog-dependent demand. IIE Trans. 41 (2009) 511-523.

[14] P. Ghare and G. Schrader, A model for exponentially decaying inventory. J. Ind. Eng. 14 (1963) $238-243$.

[15] Y. Ghiami, T. Williams and Y. Wu, A two-echelon inventory model for a deteriorating item with stock-dependent demand, partial backlogging and capacity constraints. Eur. J. Oper. Res. 231 (2013) 587-597.

[16] B. Giri, A. Goswami and K. Chaudhuri, An EOQ model for deteriorating items with time varying demand and costs. J. Oper. Res. Soc. 47 (1996) 1398-1405.

[17] A. Goswami and K. Chaudhuri, An EOQ model for deteriorating items with shortages and a linear trend in demand. J. Oper. Res. Soc. 42 (1991) 1105-1110.

[18] V. Hovelaque and L. Bironneau, The carbon-constrained EOQ model with carbon emission dependent demand. Int. J. Prod. Econ. 164 (2015) 285-291.

[19] C.-K. Huang, An integrated vendor-buyer cooperative inventory model for items with imperfect quality. Prod. Planning Control 13 (2002) 355-361.

[20] S.S. Kalantari and A.A. Taleizadeh, Mathematical modelling for determining the replenishment policy for deteriorating items in an EPQ model with multiple shipments. Int. J. Syst. Sci.: Oper. Logist. 7 (2020) 164-171.

[21] D. Khurana, S. Tayal and S. Singh, An EPQ model for deteriorating items with variable demand rate and allowable shortages. Int. J. Math. Oper. Res. 12 (2018) 117-128.

[22] D.H. Kim, A heuristic for replenishment of deteriorating items with a linear trend in demand. Int. J. Prod. Econ. 39 (1995) $265-270$.

[23] G. Mahapatra, S. Adak, T. Mandal and S. Pal, Inventory model for deteriorating items with time and reliability dependent demand and partial backorder. Int. J. Oper. Res. 29 (2017) 344-359.

[24] A. Mandelbaum and S. Zeltyn, The impact of customers' patience on delay and abandonment: some empirically-driven experiments with the $M / M / N+G$ queue. OR Spect. 26 (2004) 377-411.

[25] J. Min, Y.-W. Zhou and J. Zhao, An inventory model for deteriorating items under stock-dependent demand and two-level trade credit. Appl. Math. Modell. 34 (2010) 3273-3285.

[26] B. Pal and S. Adhikari, Price-sensitive imperfect production inventory model with exponential partial backlogging. Int. J. Syst. Sci.: Oper. Logist. 6 (2019) 27-41.

[27] S. Papachristos and K. Skouri, An optimal replenishment policy for deteriorating items with time-varying demand and partial-exponential type-backlogging. Oper. Res. Lett. 27 (2000) 175-184.

[28] S. Papachristos and K. Skouri, An inventory model with deteriorating items, quantity discount, pricing and time-dependent partial backlogging. Int. J. Prod. Econ. 83 (2003) 247-256. 
[29] D.W. Pentico, C. Toews and M.J. Drake, Approximating the EOQ with partial backordering at an exponential or rational rate by a constant or linearly changing rate. Int. J. Prod. Econ. 162 (2015) 151-159.

[30] N.H. Shah and M.K. Naik, Inventory policies for deteriorating items with time-price backlog dependent demand. Int. J. Syst. Sci.: Oper. Logist. 7 (2020) 76-89.

[31] K. Skouri, An EOQ model with backlog-dependent demand. Oper. Res. 18 (2018) 561-574.

[32] K. Skouri, I. Konstantaras, S. Papachristos and I. Ganas, Inventory models with ramp type demand rate, partial backlogging and Weibull deterioration rate. Eur. J. Oper. Res. 192 (2009) 79-92.

[33] L.M. Sloot, P.C. Verhoef and P.H. Franses, The impact of brand equity and the hedonic level of products on consumer stock-out reactions. J. Retail. 81 (2005) 15-34.

[34] E. Stavrulaki, Inventory decisions for substitutable products with stock-dependent demand. Int. J. Prod. Econ. 129 (2011) $65-78$.

[35] E. Taft, The most economical production lot. Iron Age 101 (1918) 1410-1412.

[36] J.-T. Teng and C.-T. Chang, Economic production quantity models for deteriorating items with price-and stock-dependent demand. Comput. Oper. Res. 32 (2005) 297-308.

[37] J.-T. Teng, H.-J. Chang, C.-Y. Dye and C.-H. Hung, An optimal replenishment policy for deteriorating items with time-varying demand and partial backlogging. Oper. Res. Lett. 30 (2002) 387-393.

[38] J.-T. Teng, H.-L. Yang and L.-Y. Ouyang, On an EOQ model for deteriorating items with time-varying demand and partial backlogging. J. Oper. Res. Soc. 54 (2003) 432-436.

[39] S.-P. Wang, An inventory replenishment policy for deteriorating items with shortages and partial backlogging. Comput. Oper. Res. 29 (2002) 2043-2051.

[40] H.-M. Wee, A deterministic lot-size inventory model for deteriorating items with shortages and a declining market. Comput. Oper. Res. 22 (1995) 345-356.

[41] G.A. Widyadana and H.-M. Wee, An economic production quantity model for deteriorating items with multiple production setups and rework. Int. J. Prod. Econ. 138 (2012) 62-67.

[42] K.-S. Wu, L.-Y. Ouyang and C.-T. Yang, An optimal replenishment policy for non-instantaneous deteriorating items with stock-dependent demand and partial backlogging. Int. J. Prod. Econ. 101 (2006) 369-384.

[43] C.-T. Yang, An inventory model with both stock-dependent demand rate and stock-dependent holding cost rate. Int. J. Prod. Econ. 155 (2014) 214-221.

[44] H.-L. Yang, An optimal ordering policy for deteriorating items with partial backlogging and time varying selling price and purchasing cost under inflation. Int. J. Oper. Res. 31 (2018) 403-419.

[45] P.-C. Yang and H.-M. Wee, A single-vendor and multiple-buyers production-inventory policy for a deteriorating item. Eur. J. Oper. Res. 143 (2002) 570-581.

[46] H.-L. Yang, J.-T. Yang and M.-S. Chern, An inventory model under inflation for deteriorating items with stock-dependent consumption rate and partial backlogging shortages. Int. J. Prod. Econ. 123 (2010) 8-19.

[47] S.H. Yoo, D. Kim and M.-S. Park, Economic production quantity model with imperfect-quality items, two-way imperfect inspection and sales return. Int. J. Prod. Econ. 121 (2009) 255-265. 